\title{
Nuclear Pore
}

National Cancer Institute

\section{Source}

National Cancer Institute. Nuclear Pore. NCI Thesaurus. Code C13266.

An octag onal opening, about $70 \mathrm{~nm}$ across, where the inner and outer membranes of the nuclear envelope are continuous. 